\title{
Laboratory Investigation on the Performance of Cement Stabilized Recycled Aggregate with the Vibration Mixing Process
}

\author{
Yongliang Wang $\left(D,{ }^{1}\right.$ Jinbao Zhang, ${ }^{1}$ Xuetao Wang, ${ }^{1}$ and Zhi Zhang ${ }^{2}$ \\ ${ }^{1}$ Qinghai Transportation Holding Group Co., Ltd., Xining 810001, China \\ ${ }^{2}$ Qinghai Provincial Traffic Engineering Technology Service Center, Xining 810001, China \\ Correspondence should be addressed to Yongliang Wang; wangyongliang2013@163.com
}

Received 30 October 2020; Revised 22 November 2020; Accepted 26 November 2020; Published 9 December 2020

Academic Editor: Dongyu Niu

Copyright (c) 2020 Yongliang Wang et al. This is an open access article distributed under the Creative Commons Attribution License, which permits unrestricted use, distribution, and reproduction in any medium, provided the original work is properly cited.

\begin{abstract}
The objective of this article is to investigate the effect of the vibration mixing process (VMP) on the performance of cement stabilized recycled aggregate (CSRA). The ordinary mixing process (OMP) was also used for comparative analysis with the VMP. The performance of CSRA with different cement content was tested and comprehensively analyzed by the unconfined compressive strength test, dry shrinkage test, and temperature shrinkage test. The results showed that compared with OMP, VMP can greatly improve the compressive strength, dry shrinkage, and temperature shrinkage properties of CSRA. The optimum parameters of vibration time and vibration frequency in VMP were $30 \mathrm{~s}$ and $40 \mathrm{~Hz}$, respectively. The reason why VMP can improve the performance of CSRA was that VMP strengthened the aggregate and interface transition zone and introduced microbubbles to optimize the pore structure.
\end{abstract}

\section{Introduction}

Because China's economy has developed rapidly in recent years, the existing transportation infrastructure cannot meet the increasing demand for transportation. Therefore, many roads and bridges need to be rebuilt and expanded, which will result in producing a huge amount of waste concrete. Due to the fact that the disposal of waste concrete by landfill will occupy precious land resources and damage the environment seriously, most of the waste concrete was used to produce recycled aggregate that can be directly utilized in road construction. The recycled aggregates are obtained by crushed, sieved, washed, and dried process from the demolished concrete structures. Old mortar is attached to the surface of natural aggregates, which leads to the poor performance of recycled aggregate. Compared to natural aggregate, recycled aggregate has a lower density, higher porosity, and higher water absorption. Therefore, recycled aggregates are only utilized in the low-grade road construction for road bases and surface layer of concrete in China. Cement stabilized recycled aggregate (CSRA) generally has insufficient strength and shrinkage cracking problems, which limit its application in high-grade road engineering. Currently, the recycled aggregates are strengthened before the mixing process to improve the performance of CSRA. Physical and chemical methods were proposed: (I) removing adhered mortar of coarse recycled aggregates by machine milling or soaking in acid solution and (II) filling surface pores of coarse recycled aggregates by soaking in pozzolan slurry including fly ash and silica fume. Many studies have shown that the strength process of recycled aggregate can greatly improve the mechanical properties of CSRA and improve shrinkage properties. AlBayati et al. [1] found that the water absorption of recycled aggregate was reduced by $27 \%$ with combined treatment of heat process at $350^{\circ} \mathrm{C}$ and mechanical process for $60 \mathrm{~s}$. Shaban et al. [2] soaked recycled aggregate in $40 \%$ fly ash and $3 \%$ nanosilica fume mixed slurries for $4 \mathrm{~h}$ finding that water absorption of recycled aggregate was lower by $50-55 \%$ than before and particle densities increased by $10-11 \%$. However, the strength process will greatly increase the cost and time of the production of CSRA. 
In the process of the production of CSRA, mixing is an indispensable and important process. Different mix parameters resulted in various performances of CSRA [3-5]. Han and Ferron [6] studied the relationship between mixing speed and hydration of cement pastes and found that higher speed mixing can increase the rate of cement hydration resulting in larger agglomerates. Chang and Peng [3] prepared high-performance concrete by six mixing processes and found that workability can be improved by adding all superplasticizers in water during the mixing process. Yao et al. [5] showed that the compress strength of concrete prepared by the double-blade mixer was higher than that prepared by the ordinary biaxial mixer. Feng [7] found that the vibration mixing process can make the mixture more uniform and improve the quality of the mixture. The performance of cement stabilized gravel is closely related to the degree of mixing, and the type of mixer has an important influence on the performance of the mixture $[8,9]$. Zhang et al. [10] developed a new vibrating mixing instrument for cement stabilized crushed stone, and the strength of cement stabilized crushed stone prepared by vibration mixing increased by $12.8 \%$. Li et al. [11] found that when the strength of cement stabilized gravel was the same, the cement content of cement stabilized crushed stone prepared by vibrating mixing process was lower than that of cement stabilized gravel prepared by the ordinary mixing process. Zhao et al. [12] analyzed the curve of the compressive strength of cement stabilized gravel prepared by VMP under different vibration parameters. The results showed that compared with ordinary mixing, the compressive strength of the cement stabilized gravel prepared by vibrating mixing increased and the coefficient of variation decreased. Dong et al. [13] showed that compared with ordinary mixing, vibrating mixing can improve strength, reduce water loss, and reduce shrinkage strain and the coefficient of shrinkage of cement stabilized crushed stone. These studies have shown that vibration mixing can significantly improve the performance of cement concrete. These studies show that optimization of the mixing process can significantly improve the performance of cement concrete. Compared with the traditional mixing process, vibration mixing is accompanied by mechanical vibration during mixing, which reduces the viscosity and internal friction between cementitious material and the aggregate greatly. Cement paste is easy to enter the voids and cracks on the surface of recycled aggregate and enhance the bonding force between cement paste and aggregates. It can be inferred that the cement paste can completely wrap the recycled aggregate with VMP, which will repair surface defects of recycled aggregate, such as cracks and large voids. The performance of CSRA, such as strength and shrinkage, also may be enhanced by the mixing process with vibration. However, few studies reported this.

The objective of this article is to investigate the effect of VMP on the performance of CSRA. The ordinary mixing process (OMP) was also used for comparative analysis with the vibration mixing process (VMP). Three groups of specimens with different water-cement ratios were prepared with VMP and OMP. The compressive strength, dry shrinkage, and temperature shrinkage were tested and comprehensively analyzed. The optimal parameters of VMP for CSRA were obtained by systematic analysis of experimental data. The mechanism for improving the performance of CSRA with VMP was also discussed.

\section{Materials and Testing Methods}

\subsection{Materials}

2.1.1. Cement. P.C 32.5R cement from Qinghai Qilianshan Cement Co., Ltd., was selected, and physical properties of cement were tested according to "Test Methods of Cement and Concrete for Highway Engineering” (JTG E30-2005) [14]. The test results are shown in Table 1.

2.1.2. Aggregate. Recycled coarse aggregates were provided by Huashan Road and Bridge Recycling Co., Ltd. Physical properties of coarse recycled aggregates are shown in $\mathrm{Ta}-$ ble 2. Natural fine aggregates were produced by the China Railway 17th Bureau Group Co., Ltd. Physical properties of fine natural aggregates are shown in Table 3.

2.1.3. Aggregate Gradation. According to "Technical Guidelines for Construction of Highway Roadbases" (JTG F20-2015) [15], the aggregate gradation of the CSRA is given in Figure 1.

2.2. Sample Preparation. Recycled coarse aggregates were used to completely replace natural coarse aggregates for preparing CSRA. According to "Test Method of Materials Stabilized with Inorganic Binders for Highway Engineering" (JTG E51-2009) [16], the optimum water content and maximum dry density of CSRA with a cement content of $3 \%$, $4 \%$, and $5 \%$ were tested and obtained. The experimental results of the optimum water content and maximum dry density are shown in Table 4.

The preparation of CSRA specimens was divided into four steps. At first, the quality of each component in the CSRA was weighed, including cement, coarse and fine aggregates, and the optimal content of water. Secondly, the cement, coarse and fine aggregates, and water were poured into the mixer and mixed for 2 minutes. The mixture was mixed by the double horizontal shaft vibration mixer produced by Henan Wanli Transportation Technology Group Co., Ltd. The appearance and structure of the mixer are shown in Figure 2. Vibration time and vibration frequency were considered to be important technical parameters that affect the performance of the mixture. The range of vibration time was $20 \sim 60 \mathrm{~s}$. The range of vibration frequency was 20 60 Hz. In the third step, the mixture was poured into the mold. The mold was placed on the press machine and subjected to a pressure loaded at a speed of $1 \mathrm{~mm} / \mathrm{min}$. In the fourth step, after demolding, the samples were placed in a curing chamber with a relative humidity greater than $95 \%$ at $20^{\circ} \mathrm{C}$ for 7 days. 
TABLe 1: Physical properties of cement.

\begin{tabular}{|c|c|c|c|c|c|c|c|}
\hline \multirow[t]{2}{*}{ Fineness (\%) } & \multicolumn{2}{|c|}{ Setting time $(\min )$} & \multirow[t]{2}{*}{ Volume stability $(\mathrm{mm})$} & \multicolumn{2}{|c|}{$\begin{array}{l}\text { Compressive } \\
\text { strength }(\mathrm{MPa})\end{array}$} & \multicolumn{2}{|c|}{$\begin{array}{c}\text { Flexural } \\
\text { strength } \\
(\mathrm{MPa})\end{array}$} \\
\hline & Initial setting time & Final setting time & & $3 \mathrm{~d}$ & $28 \mathrm{~d}$ & $3 \mathrm{~d}$ & $28 \mathrm{~d}$ \\
\hline 4.1 & 221 & 383 & 1.5 & 22.9 & 34.3 & 4.3 & $\overline{6.4}$ \\
\hline
\end{tabular}

TABLE 2: Physical properties of coarse aggregates.

\begin{tabular}{lccccc}
\hline $\begin{array}{l}\text { Particle } \\
\text { size } \\
(\mathrm{mm})\end{array}$ & $\begin{array}{c}\text { Specific } \\
\text { gravity } \\
\left(\mathrm{g} / \mathrm{cm}^{3}\right)\end{array}$ & $\begin{array}{c}\text { Water } \\
\text { absorption } \\
\text { rate }(\%)\end{array}$ & $\begin{array}{c}\text { Needle- } \\
\text { like } \\
\text { content } \\
(\%)\end{array}$ & $\begin{array}{c}\text { Crushed } \\
\text { value (\%) }\end{array}$ & $\begin{array}{c}\text { Mud } \\
\text { content } \\
(\%)\end{array}$ \\
\hline $5-10$ & 2.660 & 4.20 & 4.5 & - & 0.3 \\
$10-20$ & 2.636 & 2.17 & 4.3 & 24.5 & 0.2 \\
$20-30$ & 2.625 & 2.20 & 3.2 & - & 0.2 \\
\hline
\end{tabular}

TABLE 3: Physical properties of fine aggregates.

\begin{tabular}{lcc}
\hline Apparent relative density & Fineness modulus & Angularity (\%) \\
\hline 2.688 & 2.73 & 34.9
\end{tabular}

\begin{tabular}{lll}
\hline 2.688 & 2.73 & 34.9 \\
\hline
\end{tabular}

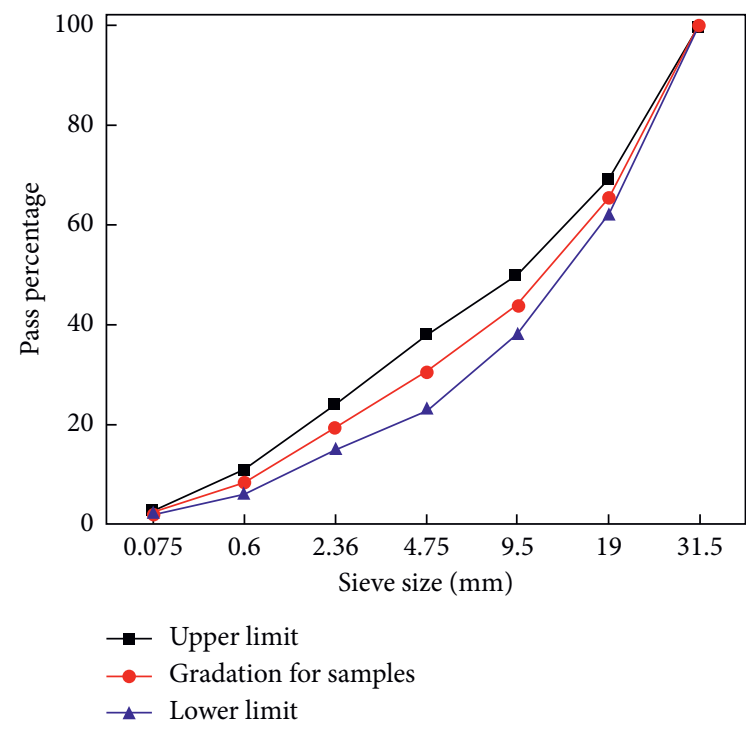

Figure 1: Gradation of CSRA.

TABLE 4: Optimum water content and maximum dry density of CSRA.

\begin{tabular}{lcc}
\hline $\begin{array}{l}\text { Cement content } \\
(\%)\end{array}$ & $\begin{array}{c}\text { Optimum water } \\
\text { content }(\%)\end{array}$ & $\begin{array}{c}\text { Maximum dry density } \\
\left(\mathrm{g} / \mathrm{cm}^{3}\right)\end{array}$ \\
\hline 3 & 6.3 & 2.236 \\
4 & 6.5 & 2.254 \\
5 & 6.8 & 2.269 \\
\hline
\end{tabular}

\subsection{Test Methods}

2.3.1. Unconfined Compressive Strength. JTG E51-2009 T0805-1994 was followed to test the unconfined compressive strength of CSRA. The mixture was molded in a standard specimen of $\Phi 150 \mathrm{~mm} \times 150 \mathrm{~mm}$ and cured with a temperature of $20 \pm 2^{\circ} \mathrm{C}$ and $95 \%$ relative humidity $(\mathrm{RH})$ for 7 days. At the given curing age, the sample was loaded at a constant loading speed of $1 \mathrm{~mm} / \mathrm{min}$ until failure. The test results were representative values of three repeated samples for each specific combination.

2.3.2. Drying Shrinkage. JTG E51-2009 T0854-2009 was followed to test the dry shrinkage of CSRA. The test sample for the drying shrinkage test was a prism measuring $100 \mathrm{~mm} \times 100 \mathrm{~mm} \times 400 \mathrm{~mm}$. The specimens were maintained in a standard curing room at a temperature of $20^{\circ} \mathrm{C} \pm 2^{\circ} \mathrm{C}$ and relative humidity above $95 \%$ for 7 days. Then, the drying shrinkage of each specimen was measured every day in the first week. After the first week, the drying shrinkage of the specimens was measured every two days. After 30 days, the drying shrinkage was recorded at $60 \mathrm{~d}$, $90 \mathrm{~d}, 120 \mathrm{~d}, 150 \mathrm{~d}$, and $180 \mathrm{~d}$. Each result was the average obtained from the testing of three specimens per mixture.

The evaluation parameter of drying shrinkage performance can be calculated as follows:

$$
\begin{aligned}
\omega_{i} & =\frac{\left(m_{i}-m_{i+1}\right)}{m_{p}}, \\
\delta_{i} & =\frac{\left(\sum_{j=1}^{4} X_{i, j}-\sum_{j=1}^{4} X_{i+1, j}\right)}{2}, \\
\varepsilon_{i} & =\frac{\delta_{i}}{l}, \\
\alpha_{d i} & =\frac{\varepsilon_{i}}{\omega_{i}}, \\
\alpha_{d} & =\frac{\sum \varepsilon_{i}}{\sum \omega_{i}},
\end{aligned}
$$

where $\omega_{i}$ is water loss rate by test time $i, \% ; \delta_{i}$ is drying shrinkage by test time $i, \mathrm{~mm} ; \varepsilon_{i}$ is dry shrinkage strain by test time $i, \% ; \alpha_{d i}$ is dry shrinkage coefficient by test time $i, \% ; \alpha_{d}$ is total dry shrinkage coefficient, $\% ; m_{i}$ is weight of specimen by test time $i, \mathrm{~g} ; X_{i, j}$ is the reading value of $j$ th dial indicator by test time $i$; $l$ is the length of the standard test specimen, $\mathrm{mm}$; and $m_{p}$ is the weight of standard test specimen after being dried, $g$.

2.3.3. Temperature Shrinkage Test. JTG E51-2009 T08552009 was followed to test the temperature shrinkage of CSRA. The temperature shrinkage test equipment was the same as the drying shrinkage test equipment. After being cured for 7 days, the sample was dried in a $105^{\circ} \mathrm{C}$ oven for 10 to 12 hours until a constant quality. The test temperature range was $40^{\circ} \mathrm{C}$ to $-10^{\circ} \mathrm{C}$, and every $10^{\circ} \mathrm{C}$ increase was used as an interval level, placing the sample in the curing chamber at 


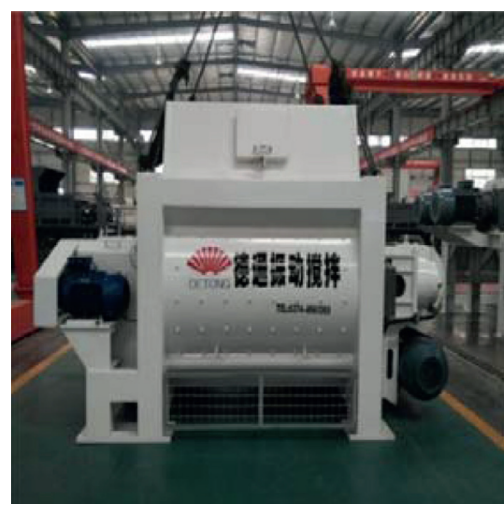

(a)

FIgURE 2: The appearance and the structure of the mixer.

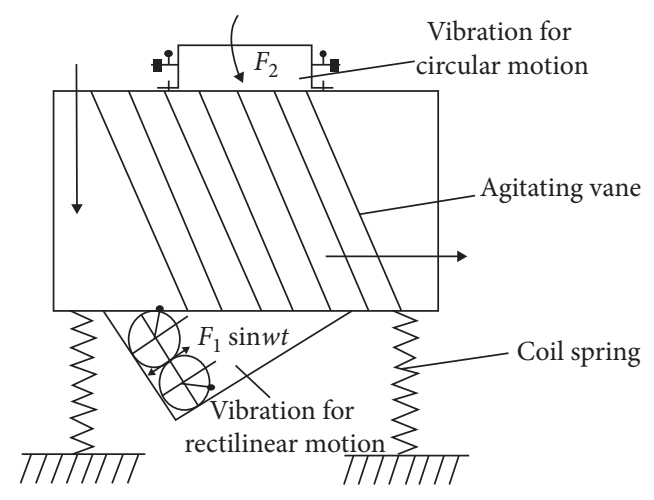

(b) the set temperature for 5 hours and then measuring the temperature shrinkage of the specimen.

The temperature shrinkage coefficient can be calculated as follows:

$$
\begin{gathered}
\varepsilon_{i}=\frac{l_{i}-l_{i+1}}{L_{0}}, \\
\alpha_{t}=\frac{\varepsilon_{i}}{t_{i}-t_{i+1}},
\end{gathered}
$$

where $l_{i}$ is the average reading value of dial indicator at the $i$ th temperature interval, $\mathrm{mm} ; t_{i}$ is the $i$ th temperature interval, ${ }^{\circ} \mathrm{C} ; L_{0}$ is the initial length of the specimen, $\mathrm{mm} ; \varepsilon_{i}$ is the average temperature shrinkage strain at the $i$ th temperature, $\%$; and $\alpha_{t}$ is the temperature shrinkage coefficient.

2.3.4. Microstructure Characteristic. The microstructure and pore structure of concrete prepared by OMP and VMP were examined. The samples were broken into small pieces and coated in gold; then, the environmental scanning electron microscope (ESEM) was used to obtain the interfacial transition zone (ITZ) of the concrete. The pore structure parameters of the concrete were determined by mercury intrusion porosimeter (MIP) at a pressure range of $135 \mathrm{kPa}$ to $415 \mathrm{MPa}$.

\section{Results and Discussion}

\subsection{Effect of Vibration Mixing Parameters on the Strength of CSRA}

3.1.1. Vibration Time. The unconfined compressive strength of the specimen was tested after 7 days of curing. The test results of CSRA with different cement content prepared with a vibration frequency of $40 \mathrm{~Hz}$ and vibration times of $20 \mathrm{~s}$, 30 s, 40 s, 50 s, and 60 s are shown in Figure 3. As Figure 3 depicted, the cement content determines the compressive strength of CSRA. The unconfined compressive strength of CSRA increases with the increase of cement content. VMP was beneficial to improve the mechanical strength of CSRA. As the vibration time increases, the compressive strength increases. From the relationship curve between vibration time and compressive strength, it is found that there is an optimal vibration time. When the vibration time is $30 \mathrm{~s}$, the compressive strength has a significant increase. When the vibration time is $30 \mathrm{~s}$ and the vibration frequency is $40 \mathrm{~Hz}$, the unconfined compressive strength of CSRA with $3 \%$ cement content is $3.0 \mathrm{MPa}$. It meets the road base strength requirements for medium and light traffic in China.

3.1.2. Vibration Frequency. The unconfined compressive strength of CSRA with different cement content prepared with vibration time of $30 \mathrm{~s}$ and vibration frequency of $20 \mathrm{~Hz}$, $30 \mathrm{~Hz}, 40 \mathrm{~Hz}, 50 \mathrm{~Hz}$, and $60 \mathrm{~Hz}$ are shown in Figure 4. It is demonstrated in Figure 4 that the cement content determines the compress strength of CSRA, which is consistent with the conclusion of Section 3.1.1. As the vibration frequency increases, the unconfined compressive strength first increases and then decreases. From the relationship curve between vibration frequency and compressive strength, it is found that $40 \mathrm{~Hz}$ is the optimal vibration frequency. When the vibration time is $30 \mathrm{~s}$ and the vibration frequency is $40 \mathrm{~Hz}$, the unconfined compressive strength of CSRA with $5 \%$ cement content is $4.2 \mathrm{MPa}$. It meets the road base strength requirements for medium and light traffic in China.

\subsection{Effect of VMP on the Performance of CSRA}

3.2.1. Compressive Strength. The compressive strength of CSRA prepared by VMP was tested to compare with the compressive strength of CSRA prepared by OMP. Figure 5 shows the compressive strength of CSRA prepared by VMP and OMP at different ages. It can be seen from Figure 5 that compared with OMP, the compressive strength of CSRA was improved by VMP. When the cement content is 3\%, 7-day compressive strength and 28-day compressive strength increase by $15.4 \%$ and $9.7 \%$, respectively. When the cement content is $4 \%$, the compressive strength of 7 days and 28 days increases by $16.2 \%$ and $9.6 \%$, respectively. However, when the cement content is $5 \%$, the compressive strength of 7 days and 28 days only increases by $4.5 \%$ and $5.1 \%$. It indicates that the additional energy of vibration can destroy the cement agglomeration structure, and the cement is evenly dispersed into smaller cement particles, resulting in cement hydration 


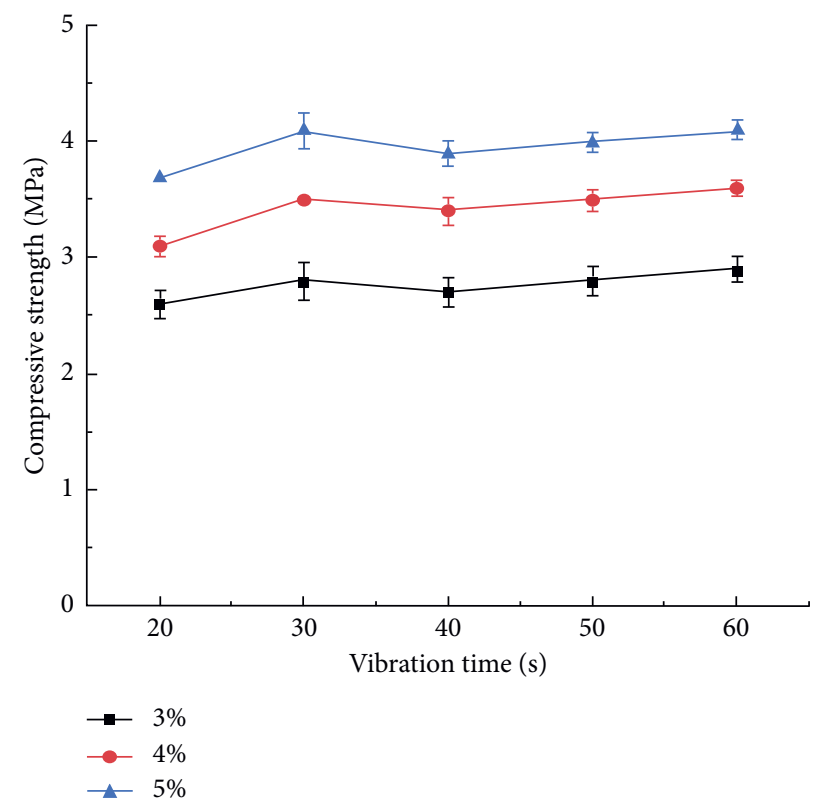

FIgURE 3: Seven-day compressive strength of CSRA with different vibration times.

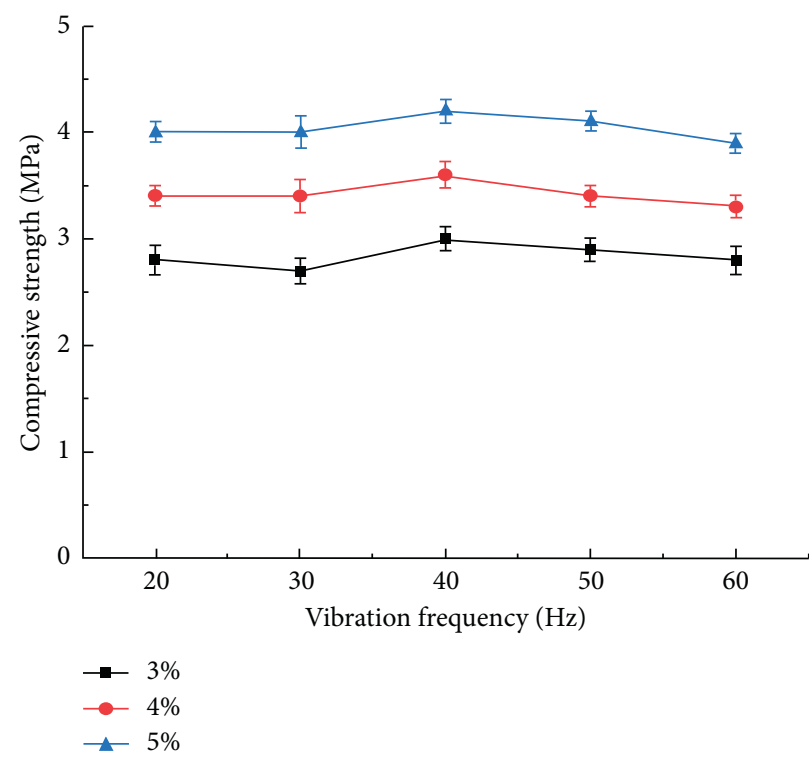

FIGURE 4: Seven-day unconfined compressive strength of CSRA with different vibration frequencies.

reaction more sufficient [17]. The smaller the cement content in CSRA, the more obvious this improvement effect of VMP. Figure 6 shows the micromorphology of the interface transition zone CSRA prepared by OMP and VMP. Compared with the specimen prepared by nonvibration mixing, the specimen prepared by vibrating mixing has a tighter bond and higher bonding strength between cement paste and recycle aggregate. Better interface transition zone results in higher compressive strength of cement stabilized recycle aggregate [18]. During the VMP, the collision of recycled aggregates will also strengthen the recycled aggregates, which is also beneficial to the improvement of the compressive strength of CSRA.
3.2.2. Drying Shrinkage. The drying shrinkage of CSRA prepared by VMP and OMP at different ages are shown in Figures 7-9. As shown from Figures 7-9 with the two mixing methods, as the age increases, the cumulative water loss rate and dry shrinkage strain of CSRA increase for all specimens. When the cement dosage is the same, the cumulative water loss rate, dry shrinkage strain, and dry shrinkage coefficient of vibration mixed CSRA are lower than those of ordinary mixed CSRA. As cement content increases, the drying shrinkage coefficient of CSRA increases. Although the increase in cement content is good for compressive strength, it is not good for shrinkage performance. It is worth noting that both strength and the drying shrinkage can be improved by VMP. 


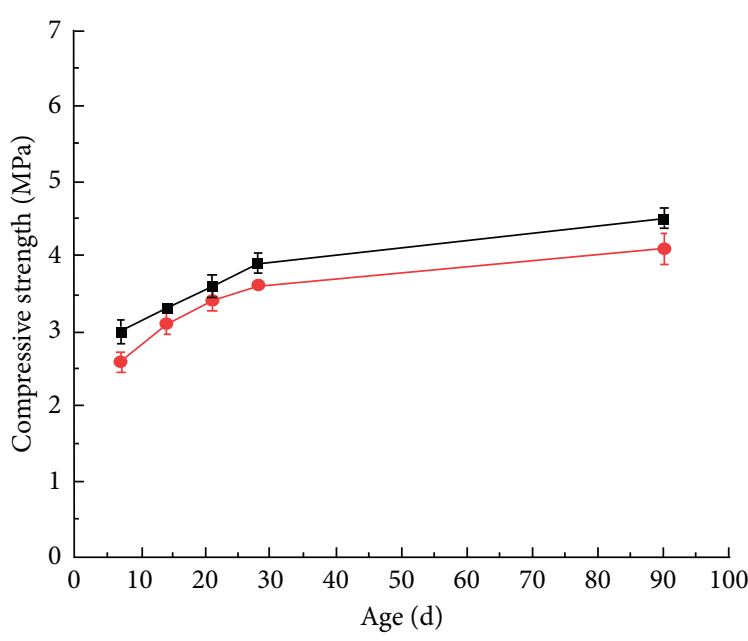

$\rightarrow$ Vibration mixing process $\rightarrow$ Ordinary mixing process

(a)

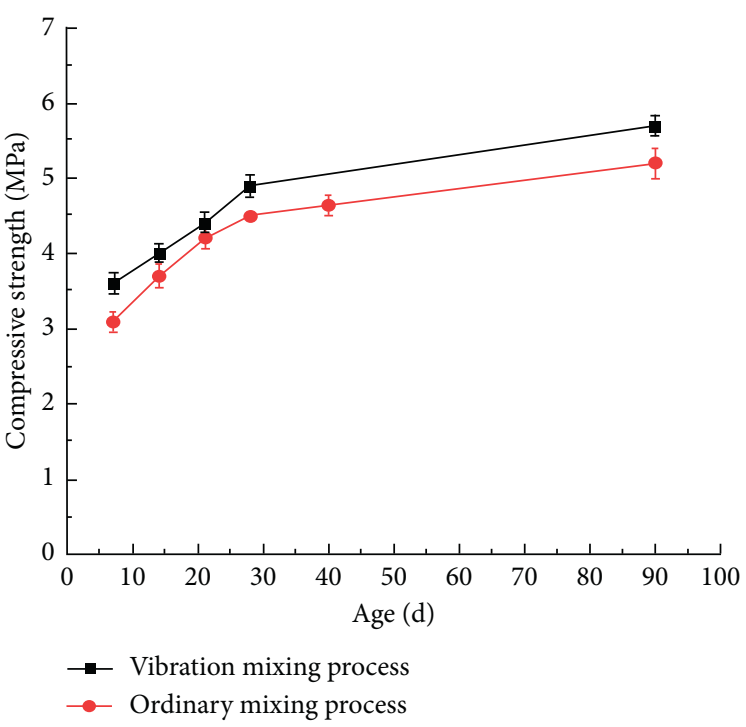

(b)

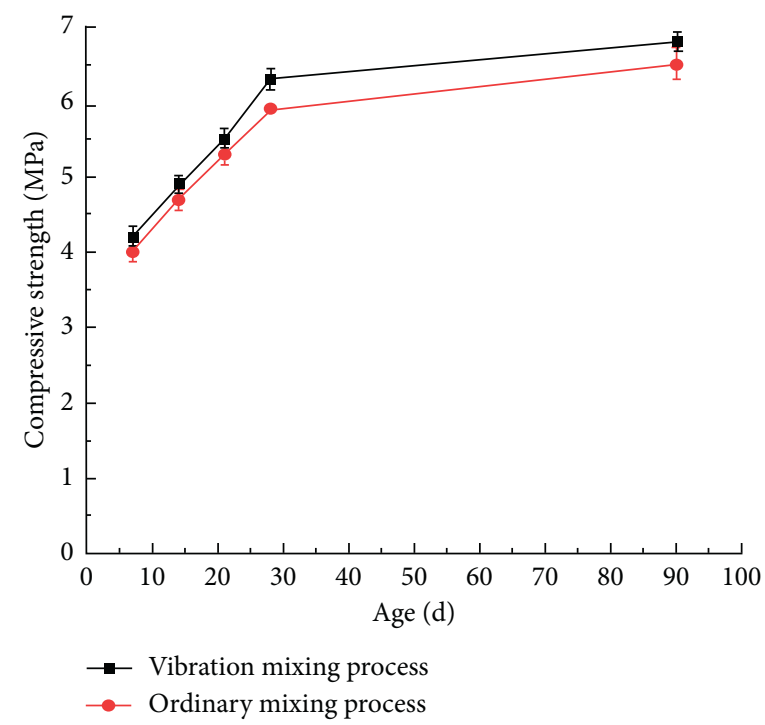

(c)

Figure 5: Compressive strength of CSRA with different cement contents. (a) 3\%. (b) $4 \%$. (c) 5\%.

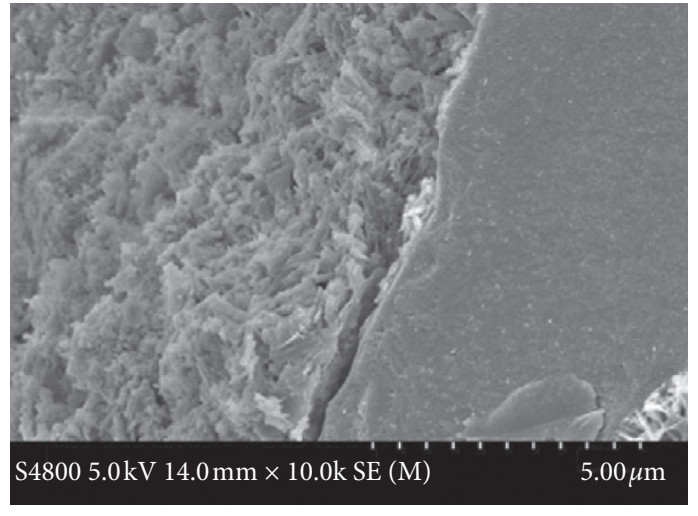

(a)

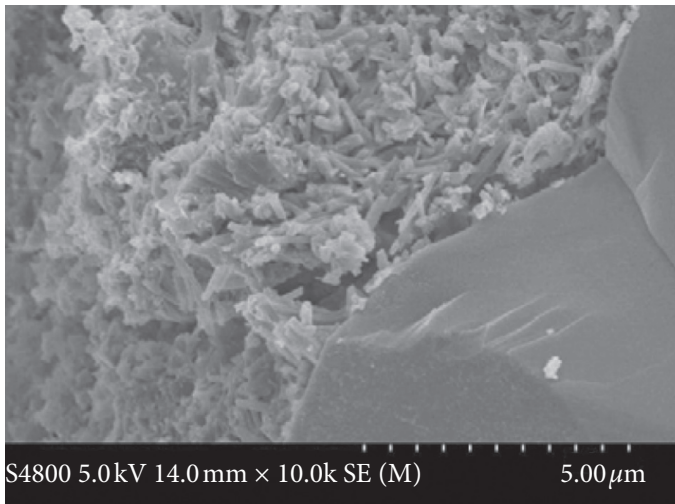

(b)

FIGURE 6: Micromorphology of the interface transition zone of CSRA. (a) OMP. (b) VMP. 


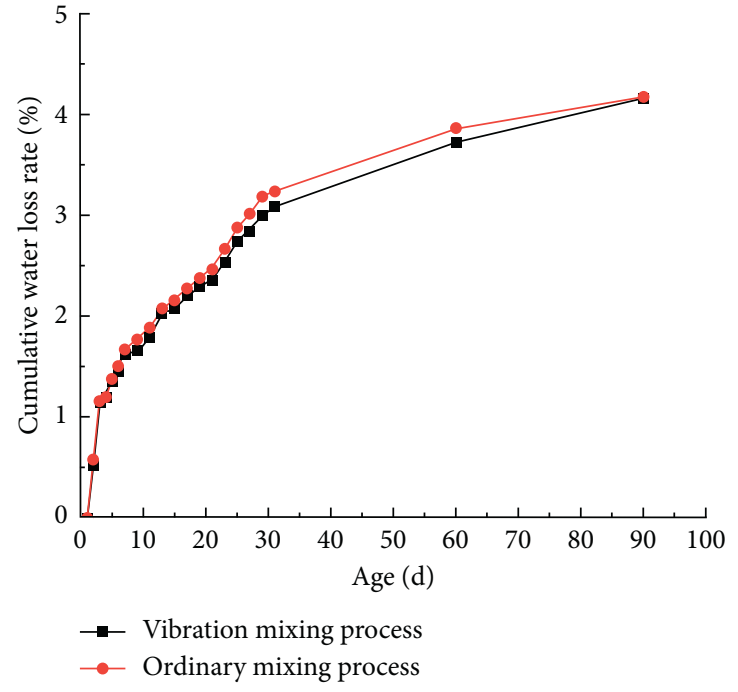

(a)

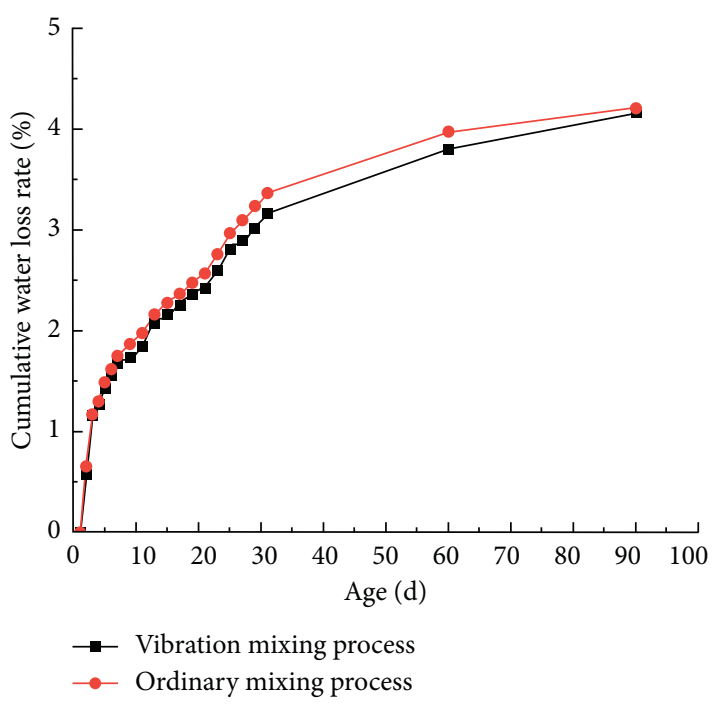

(b)

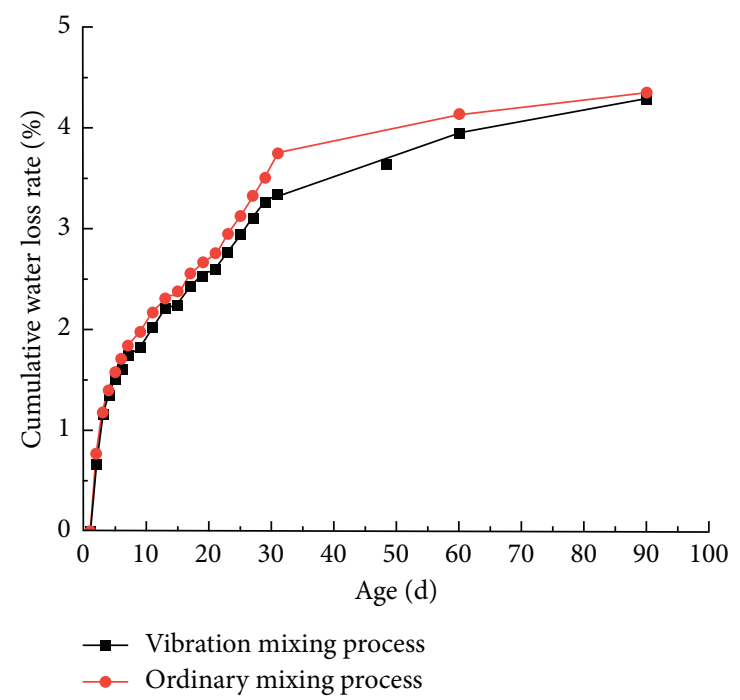

(c)

Figure 7: Cumulative water loss rate of CSRA with different cement contents. (a) $3 \%$. (b) $4 \%$. (c) $5 \%$.

Table 5 shows the pore structure parameters of CSRA with $4 \%$ cement content prepared by OMP and VMP. The values of pore structure parameters including pore surface area, area median aperture, average aperture, and porosity of the CSRA with vibration mixing are all smaller than those of the CSRA without vibration mixing. This shows that vibration mixing can reduce the number of large pores, reduce the pore size, and optimize the structure of the pores. The increase of porosity indicates that the vibration mixing process has a proper airentraining effect. When the CSRA is mixed with vibration, the water film on the surface of the aggregate and the cement particles is broken, so that more air can be introduced. At the same time, the large bubbles are broken due to the vibration, which optimizes the pore structure of CSRA [19]. Loss of capillary water will cause dry shrinkage of the CSRA, which will lead to increased tensile stress. The VMP introduces a large number of tiny bubbles to close the capillary structure to prevent the evaporation of water and improve the shrinkage performance of CSRA.

3.2.3. Temperature Shrinkage. The temperature shrinkage test results of CSRA with different cement content are shown in Figure 10. As Figure 10 depicts, compared with OMP, VMP can improve the temperature shrinkage of CSRA greatly. At the same cement content, the temperature shrinkage coefficient of CSRA prepared by VMP is lower than that of CSRA prepared by OMP. Similar to the development curve of drying shrinkage, the temperature shrinkage coefficient increases with the increase of cement content. Compared with OMP, when the temperature range is $30^{\circ} \mathrm{C} \sim 20^{\circ} \mathrm{C}$ and the cement content is $5 \%$, the temperature shrinkage coefficient of the stabilized recycled aggregate of vibration mixing cement is reduced by about $27 \%$. The 


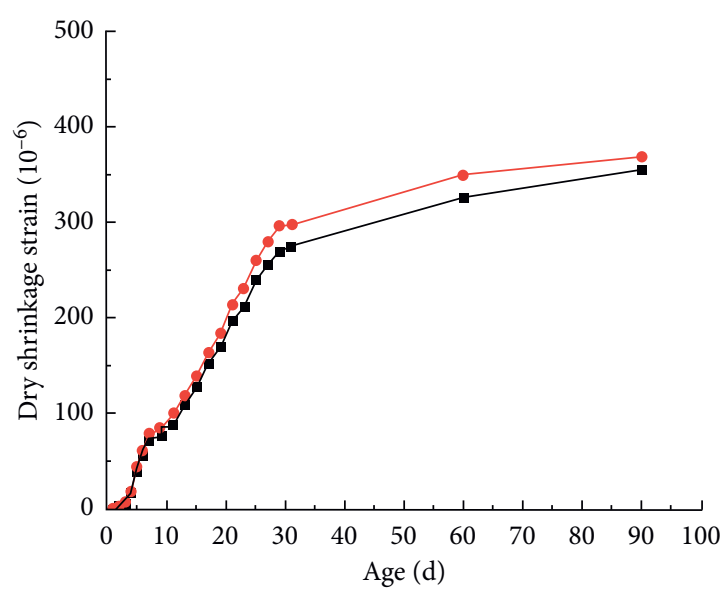

$\rightarrow-$ Vibration mixing process $\multimap$ Ordinary mixing process

(a)

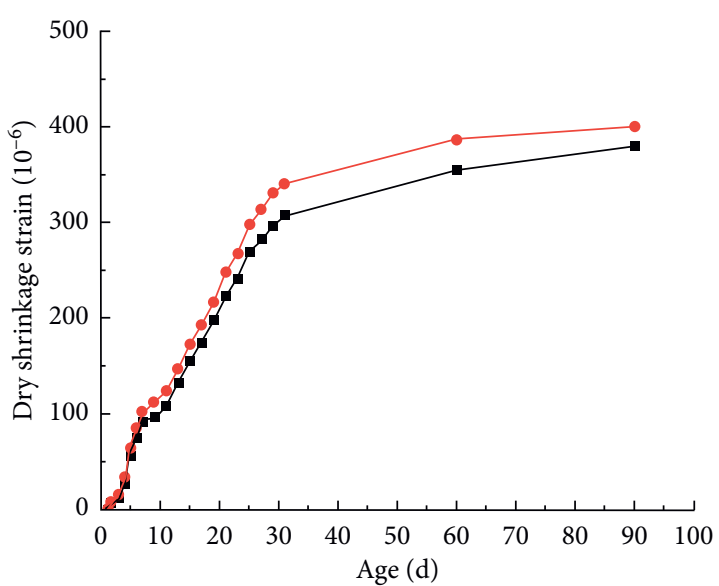

- Vibration mixing process

$\multimap$ Ordinary mixing process

(b)

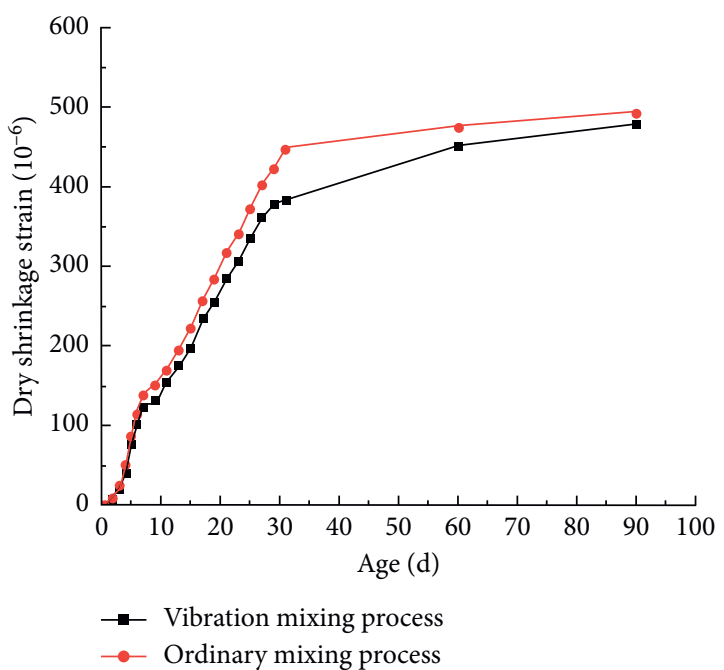

(c)

FIGURE 8: Dry shrinkage strain of CSRA with different cement contents. (a) $3 \%$. (b) $4 \%$. (c) $5 \%$.

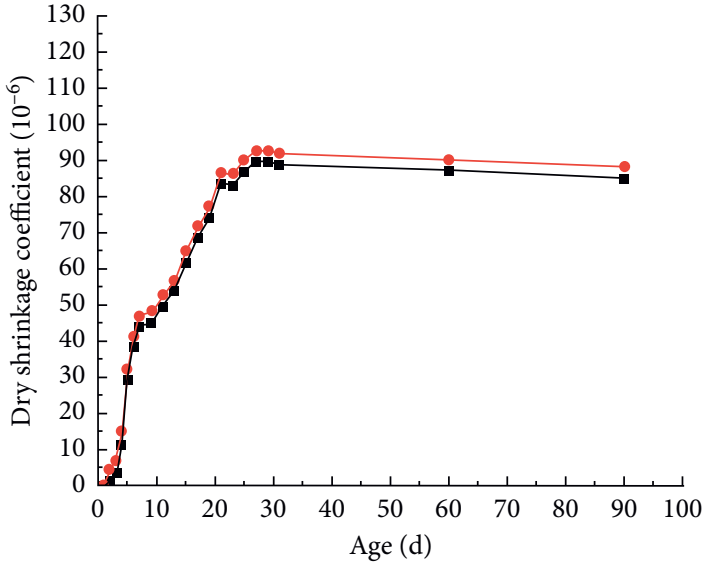

- Vibration mixing process

$\multimap$ Ordinary mixing process

(a)

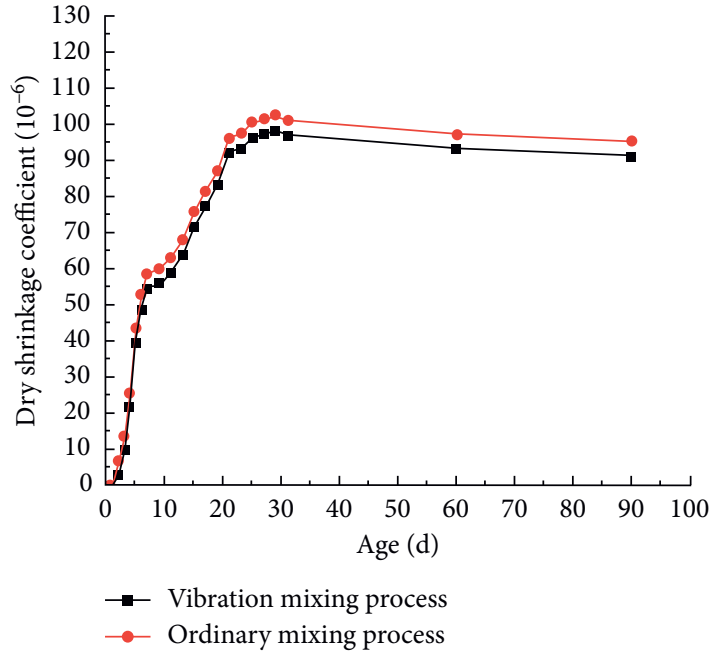

(b)

Figure 9: Continued. 


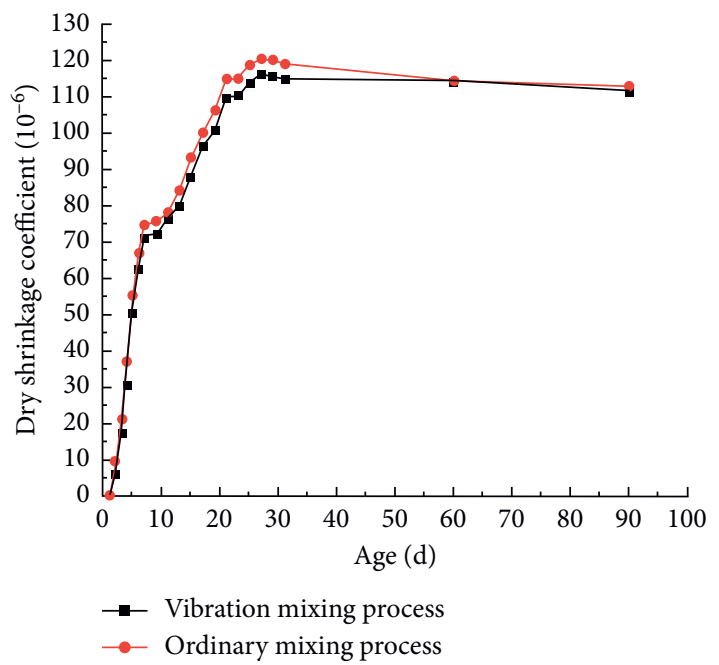

(c)

FIGURE 9: Dry shrinkage coefficient of CSRA with different cement contents. (a) $3 \%$. (b) 4\%. (c) $5 \%$.

TABLE 5: The pore structure parameters of CSRA with $4 \%$ cement content.

\begin{tabular}{lcc}
\hline Parameters & Vibration mixing process & Ordinary mixing process \\
\hline Pore surface area $\left(\mathrm{m}^{2} / \mathrm{g}\right)$ & 2.712 & 4.359 \\
Area median aperture $(\mu \mathrm{m})$ & 0.315 & 0.454 \\
Average aperture $(\mu \mathrm{m})$ & 0.048 & 0.059 \\
Porosity $(\%)$ & 16.01 & 12.21 \\
\hline
\end{tabular}

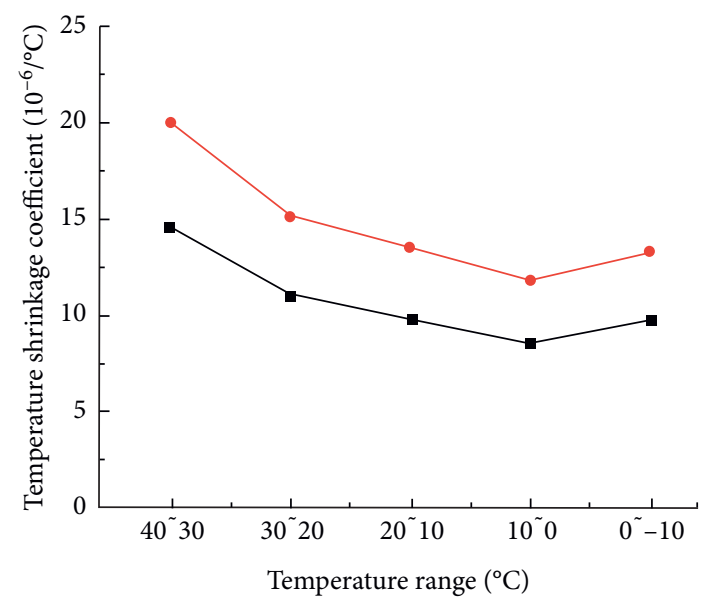

- Vibration mixing process

$\longrightarrow$ Ordinary mixing process

(a)

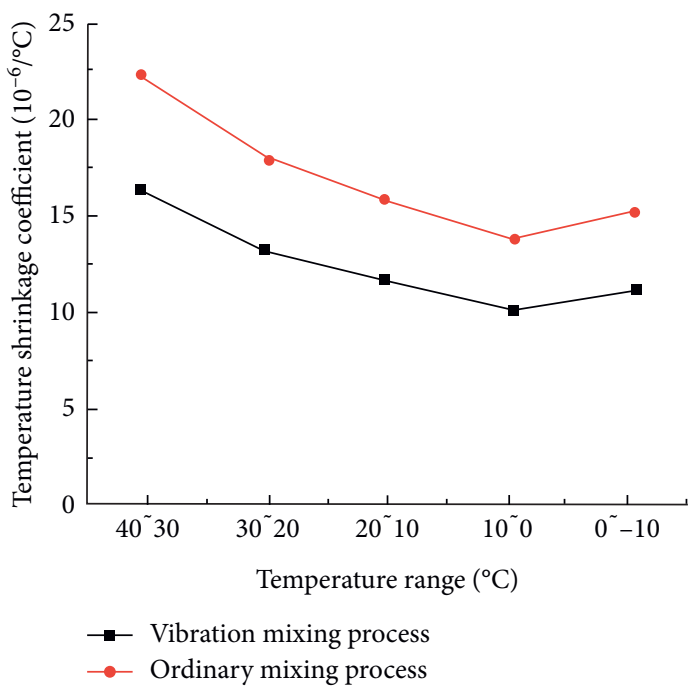

(b)

FIgURe 10: Continued. 


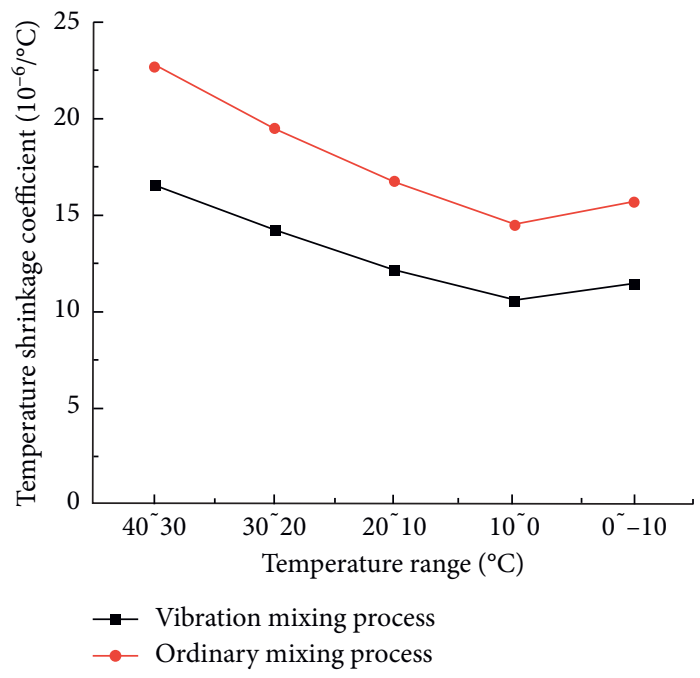

(c)

FIgURE 10: Temperature shrinkage coefficient of CSRA with different cement contents. (a) $3 \%$. (b) $4 \%$. (c) $5 \%$.

temperature shrinkage cracks of CSRA refer to the cracks caused by the volume shrinkage caused by the distance between the molecules of the internal structure of the mixture as the temperature changes. Through VMP, the homogeneity of the mixture is improved and the interface transition zone leads to the improvement of temperature shrinkage performance [20].

\section{Conclusions}

(1) Compared with OMP, VMP can greatly improve the compressive strength, dry shrinkage, and temperature shrinkage properties of CSRA.

(2) Considering the road performance and economic benefits, the optimum parameters of vibration time and vibration frequency in VMP were $30 \mathrm{~s}$ and $40 \mathrm{~Hz}$, respectively.

(3) The reason why VMP can improve the performance of CSRA was that VMP strengthened the aggregate and interface transition area and introduced microbubbles to optimize the pore structure.

\section{Data Availability}

The data used to support the findings of this study are included within the article.

\section{Conflicts of Interest}

The authors declare that they have no conflicts of interest.

\section{Acknowledgments}

This study was supported by Science and Technology Department Project of Qing Hai Province (2021-QY-216) and Transportation Department Project of Qing Hai Province (2019-14).

\section{References}

[1] H. K. A. Al-Bayati, P. K. Das, S. L. Tighe, and H. Baaj, "Evaluation of various treatment methods for enhancing the physical and morphological properties of coarse recycled concrete aggregate," Construction and Building Materials, vol. 112, pp. 284-298, 2016.

[2] W. M. Shaban, J. Yang, H. Su et al., "Properties of recycled concrete aggregates strengthened by different types of pozzolan slurry," Construction and Building Materials, vol. 216, pp. 632-647, 2019.

[3] P.-K. Chang and Y.-N. Peng, "Influence of mixing techniques on properties of high performance concrete," Cement and Concrete Research, vol. 31, no. 1, pp. 87-95, 2001.

[4] T. Hemalatha, K. R. Ram Sundar, A. R. Murthy, and $\mathrm{N}$. R. Iyer, "Influence of mixing protocol on fresh and hardened properties of self-compacting concrete," Construction and Building Materials, vol. 98, pp. 119-127, 2015.

[5] Y. Yao, Z. Feng, S. Chen, B. Li, L. Zhao, and W. Zhao, "A double-blade mixer for concrete with improved mixing quality," Arabian Journal for Science and Engineering, vol. 41, pp. 4809-4816, 2016.

[6] D. Han and R. D. Ferron, "Influence of high mixing intensity on rheology, hydration, and microstructure of fresh state cement paste," Cement and Concrete Research, vol. 84, pp. 95-106, 2016.

[7] Z. Feng, W. Wang, L. Zhao et al., "Test research on concrete vibratory mixing techniques," in Proceedings of the 2006 Xi'an International Conference of Architecture and Technology, pp. 825-830, Lodz, Poland, April 2006.

[8] C. F. Ferraris, "Concrete mixing methods and concrete mixers: state of the art," Journal of Research of the National Institute of Standards and Technology, vol. 106, no. 2, pp. 391-399, 2001.

[9] J. Dils, G. De Schutter, and V. Boel, "Influence of mixing procedure and mixer type on fresh and hardened properties of concrete: a review," Materials and Structures, vol. 45, no. 11, pp. 1673-1683, 2012.

[10] H. Zhang, S. Liang, H. Yang, and L. Yul, "Research on performance of cement stabilized crushed stone based on indoor 
vibration stirring," Journal of China Highway, vol. 31, pp. 58-65, 2018.

[11] R. Li, S. Luo, H. Wang, Z. Guang, and M. Liu, "Comparative study on the strength performance of vibrating agitated cement stabilized gravel," Journal of Chongqing Jiaotong University (Natural Science Edition), vol. 36, pp. 33-36, 2017.

[12] L. Zhao, W. Jiang, J. Hou, Z. Feng, and S. Xue, "Influence of mixing methods on performance of compressive strength for cement stabilized Macadam mixture," Journal of China Highway, vol. 31, pp. 151-158, 2018.

[13] W. Dong, S. Xie, L. Zhao, and Z. Feng, "Influence of vibration on mixing process of cement stabilized Macadam and its performance," Journal of China Highway, vol. 32, pp. 181-190, 2019.

[14] JTG E30-2005, Highway Engineering Cement and Cement Concrete Test Specification, China Communication Press, Beijing, China, 2005.

[15] JTG F20-2015, Technical Guidelines for Construction of Highway Roadbases, China Communication Press, Beijing, China, 2015.

[16] JTG E51-2009, Test Methods of Materials Stabilized with Inorganic Binders for Highway Engineering, China Communication Press, Beijing, China, 2009.

[17] G. Xiong, C. Wang, S. Zhou et al., "Preparation of high strength lightweight aggregate concrete with the vibration mixing process," Construction and Building Materials, vol. 229, p. 116936, 2019.

[18] P. Vargas, O. Restrepo-Baena, and J. I. Tobón, "Microstructural analysis of interfacial transition zone (ITZ) and its impact on the compressive strength of lightweight concretes," Construction and Building Materials, vol. 137, pp. 381-389, 2017.

[19] J. Zhang, X. Gao, and L. Yu, "Improvement of viscositymodifying agents on air-void system of vibrated concrete," Construction and Building Materials, vol. 239, Article ID 117843, 2020.

[20] V. Collin and P.-H. Jézéquel, "Mixing of concrete or mortars: distributive aspects," Cement and Concrete Research, vol. 39, no. 8, pp. 678-686, 2009. 